\title{
Actividad eléctrica cerebral de la atención en adolescentes policonsumidores por medio de un equipo de BCI (Brain Control Interface)
}

\author{
ANGIE ALEJANDRA DÍAZ BAQUERO* \\ Universidad Católica de Colombia \\ ORCID: https://orcid.org/0000-0002-0815-4480 \\ SANDRA MILENA CAMELO ROA** \\ Universidad Católica de Colombia \\ ORCID: https://orcid.org/0000-0002-5983-075X
}

\begin{abstract}
How to quote this article: Díaz Baquero, A.A. \& Camelo Roa, S.M. (2019). Actividad eléctrica cerebral de la atención en adolescentes policonsumidores usando un equipo BCI (brain control interface). Acta Colombiana de Psicología, 22(1), 175-188. doi: http://www.dx.doi.org/10.14718/ ACP.2019.22.1.9
\end{abstract}

Resumen

\begin{abstract}
El consumo de sustancias psicoactivas es un problema de salud pública que afecta cada vez más a la población adolescente. La presente investigación tuvo como objetivo registrar la actividad eléctrica cerebral (EEG) en tareas de atención (sostenida y selectiva) en un grupo de adolescentes policonsumidores. Se empleó un diseño ex post-facto retrospectivo con grupo cuasi control, en 46 adolescentes con edades entre los 12 los 17 años: 23 policonsumidores y 23 cuasi-controles. Para el registro de la actividad eléctrica cerebral se utilizó un equipo de BCI (Brain Control Interface) Emotiv EPOC research grade 14 Channel Mobile EEG y se aplicó el Programa virtual de entrenamiento cerebral Brain HQ con el módulo "enfoco mi atención" para la evaluación de la atención. Los resultados mostraron un incremento de ondas cerebrales beta- $\beta$ (13-30 Hz), theta- $\theta$ (4-7 Hz) y delta- $\delta(3-4 \mathrm{~Hz})$ en áreas frontales y prefrontales en los adolescentes policonsumidores en tareas de atención en comparación con el grupo cuasi-control. Se identificó una diferencia significativa con respecto al tiempo de respuesta entre los adolescentes consumidores de sustancias psicoactivas frente al grupo cuasi-control en ambos tipos de tareas atencionales.
\end{abstract}

Palabras clave: adolescentes, atención, BCI (Brain Control Interface), electroencefalografía, Emotiv EPOC, policonsumo.

\section{Brain electrical activity of attention in polydrug adolescents using an equipment BCI (Brain Control Interface)}

\begin{abstract}
The consumption of psychoactive substances is a public health problem that increasingly affects the adolescent population. This investigation had the objective of record the brain electrical activity (EEG) in attention tasks (sustained and selective) in a group of polyconsumers. Employment a retrospective ex post-facto design with a quasi-control group with 46 adolescents between 12-17 years old: 23 polyconsumers and 23 quasi-controls. For the recording of brain electrical activity, it was used a equipment BCI (Brain Control Interface) research grade 14 Channel Mobile EEG and applied the Brain Training Virtual Program "Brain HQ" module "focus my attention" to evaluate the attention. The results showed an increase in beta- $\beta$ (13$30 \mathrm{~Hz})$, theta- $\theta(4-7 \mathrm{~Hz})$ and delta- $\delta(3-4 \mathrm{~Hz})$ brain waves in frontal and prefrontal areas in adolescent polyonsumers versus the quasi-control group in attention tasks. Likewise, identified a significant difference with respect to the response time between adolescents consuming psychoactive substances in relation to the quasi-control group in both types of attentional tasks. Keywords: attention, BCI (Brain Control Interface), electroencephalography, emotiv EPOC, polydrug, teenagers.
\end{abstract}

\footnotetext{
* Avenida Caracas \# 46-72, Bogotá, Colombia, (57) 3277300-50-62. Joven Investigador Colciencias 2016, aadiaz93@ucatolica.edu.co ** Avenida Caracas \# 46-72, Bogotá, Colombia, (57) 3277300-50-62. Dic. Línea de Investigación en Procesos Psicobiológicos. smcamelo@catolica.edu.co

Artículo de investigación Joven Investigador financiado por el Departamento Administrativo de Ciencia, Tecnología e Investigación (Colciencias) y la Facultad de Psicología de la Universidad Católica de Colombia, bajo la convocatoria 645.
} 


\title{
Atividade elétrica cerebral da atençáo em adolescentes policonsumidores por meio de um equipamento de Brain Control Interface (BCI)
}

Resumo

\begin{abstract}
O consumo de substâncias psicoativas é um problema de saúde pública que afeta cada vez mais a população adolescente. Esta pesquisa teve como objetivo registrar a atividade elétrica cerebral (EEG) em tarefas de atenção (sustentada e alternada) num grupo de adolescentes policonsumidores. Foi empregado um desenho ex post-facto retrospectivo com grupo quase- controle, em en 46 adolescentes entre 12 e 17 anos de idade: 23 policonsumidores e 23 quase-controles. Para o registro da atividade elétrica cerebral, foi utilizado um equipamento de Brain Control Interface (BCI) Emotiv EPOC research grade 14 Channel Mobile EEG e foi aplicado o Programa Virtual de Treinamento Cerebral Brain HQ, com o módulo "foco minha atenção" para a avaliar a atenção. Os resultados mostraram um aumento de ondas cerebrais beta- $\beta(13-30 \mathrm{~Hz})$, theta- $\theta(4-7 \mathrm{~Hz})$ e delta- $\delta$ (3-4 Hz) em áreas frontais e pré-frontais nos adolescentes policonsumidores em tarefas de atenção em comparação com o grupo quase-controle. Foi identificada uma diferença significativa a respeito do tempo de resposta entre os adolescentes consumidores de substâncias psicoativas ante o grupo quase-controle em ambos os tipos de tarefas de atenção.

Palavras-chave: adolescentes, atenção, BCI (Brain Control Interface), eletroencefalografia, Emotiv EPOC, policonsumo.
\end{abstract}

\section{Introducción}

De acuerdo con el Fondo de las Naciones Unidas para la Infancia (UNICEF, 2011), la adolescencia se define como una etapa que trascurre entre los 10 y 19 años de edad, caracterizada por cambios emocionales, cognitivos, conductuales y fisiológicos significativos. Al respecto, en la literatura se reconocen múltiples diferencias neuroanatómicas (Golarai et al., 2007; Mota \& Corral, 2011), neurobioquímicas (López-Caneda et al., 2014) y neurofisiológicas (Weise, Eisenhofer \& Merke, 2002) que producen cambios fisiológicos y en los mecanismos cognoscitivos propios de la inhibición y la búsqueda de recompensa en los adolescentes (Lacono, Malone \& McGue, 2008), lo que, en conjunto, genera mayor vulnerabilidad para la asunción de conductas de riesgo, como sucede con el consumo de sustancias psicoactivas (Corominas, Roncero, Bruguera \& Casas, 2007; Garcia, Garcia \& Rivera, 2015).

Teniendo esto en cuenta, el Estudio Nacional de Consumo de Sustancias Psicoactivas en Colombia realizado por la UNICEF (2013) señaló que el consumo de alcohol y tabaco es el de mayor predominio en el país y que la sustancia más consumida por jóvenes entre los 12 y 17 años es la marihuana. Asimismo, el Gobierno Nacional de la República de Colombia (2013), con el apoyo de la Oficina de Naciones Unidas contra la Droga y el Delito (UNODC), la Comisión Interamericana para el Control del Abuso de Drogas (CICAD) de la Organización de los Estados Americanos (OEA) y la Embajada de los Estados Unidos en Colombia (INL), indicó que el $13 \%$ de los encuestados en Colombia había consumido alguna sustancia ilícita al menos una vez en su vida; que la sustancia más consumida es el cannabis, seguida por la cocaína; y que, del grupo de encuestados, el $62 \%$ correspondía a adolescentes con edades entre los 12 y 24 años.

De igual forma, en el 2016, la UNODC (2016) indicó que 250 millones de personas con edades comprendidas entre los 15 y 64 años consumieron al menos una sustancia ilícita durante el 2014, que 29 millones de consumidores padecieron algún trastorno asociado con dicho consumo, $\mathrm{y}$ que hubo un aumento del consumo de cocaína de 14 millones a 18.8 millones en el 2014, además de un mantenimiento del consumo de cannabis (del 3.8 \%) entre los años 1998 y 2014, entre otros hallazgos importantes.

El consumo de sustancias psicoactivas se entiende desde el DSM-IV como la dependencia a diversas sustancias que experimenta un individuo, y se caracteriza por ser un consumo de por lo menos tres sustancias (no se incluye cafeína o nicotina) durante un periodo de al menos 12 meses (APA, 2005). En esta misma línea, el craving se define como el deseo intenso de consumir una o varias sustancias psicoactivas, y este se presenta durante el periodo de abstinencia en los individuos farmacodependientes, debido a determinados mecanismos de aprendizaje en los que se incita el deseo a partir de un estímulo presente en el ambiente que ha sido asociado anteriormente con el consumo de sustancias (Acosta et al., 2011).

Desde la neurobiología, la adicción y el craving tras el policonsumo se explican al identificar la dopamina como el neurotransmisor con mayor implicación, ya que, como afirma Redolar (2008), este neurobioquímico es una de las catecolaminas más importantes del sistema nervioso 
central implicadas en el reforzamiento de procesos de aprendizaje y memoria. De acuerdo con Daza (2009), en la adolescencia se observa una disminución de la función del sistema dopaminérgico mesolímbico que produce una reducción de dopamina en la hendidura sináptica, lo que contribuye con cambios emocionales y conductuales, ya que se evidencia una disminución del nivel de motivación que causa alteraciones conductuales significativas y características de esta etapa -que, en general, giran alrededor del aburrimiento, la insatisfacción e incluso la anhedonia-. Estos cambios neurobioquimicos contribuyen a la explicación de la incurrencia de los adolescentes en conductas reforzantes, como el consumo de sustancias.

Al revisar la literatura, es posible identificar diversos estudios que muestran los cambios que generan las sustancias psicoactivas a nivel neurobiológico y que alteran la neurobioquímica y la fisiología cerebral tanto en adolescentes como en adultos. Específicamente, se han encontrado estudios que identifican estos cambios con el alcohol (Esel, 2006; Fagundo, Martin-Santos, Abanades, Farré \& Verdejo-García, 2008; García \& Barriguete, 2012; Gilpin \& Koob, 2008; León et al., 2014; López-Caneda et al. 2014), la cocaína (Caballero, 2005; Connolly, Foxe, Nierenberg, Shpaner \& Garavan, 2012; Crespo-Fernández \& Rodríguez, 2007; Fernández-Espejo, 2006; Guardia, 2001; Madoz-Gúríde, Ochoa \& Martínez, 2009; Moratalla, 2008; National Intitute on Drug Abuse, 2010; Nestler, 2005; Urigüen \& Callado, 2010), el éxtasis (Colado, 2008; Gaviria, 2010; Partilla et al., 2006), la heroína (Kosten \& George, 2002; Kreek et al., 2012), la nicotina (D’Souza \& Markou, 2011; Pérez-Rubio et al., 2011) y el cannabis (Mena et al., 2013; Rodríguez, Carrillo \& Soto, 2005; van Hell et al., 2011).

\section{Atención y electroencefalografía en policonsumidores}

La atención es definida como un conjunto coordinado de mecanismos que cumplen una función en la identificación y selección de estímulos ambientales importantes para la resolución de tareas cognoscitivas (Ríos, Muñoz \& Paúl, 2007). Dentro de la clasificación de los diferentes subtipos de atención, se identifican dos clases: (a) la atención selectiva, que hace referencia a la capacidad para centrarse en un estímulo relevante del entorno y eliminar aquellos que no son relevantes en un determinado momento; y (b) la atención sostenida, que se entiende como la capacidad para mantener la atención focalizada en la realización de una tarea durante tiempos prolongados (Sánchez, Vázquez \& Valiente, 2011).

Con respecto a esto, se ha encontrado la presencia de un déficit de atención en adolescentes policonsumidores que coexiste con alteraciones psicofisiológicas, debido a que el consumo de distintas sustancias psicoactivas produce alteraciones sobre la actividad cerebral normal y provoca variaciones con respecto a la frecuencia y amplitud de las ondas cerebrales alrededor de distintas áreas del cerebro (Acosta, Cervantes \& Puentes, 2009; Colzato, van den Wildenberg \& Hommel, 2009; Coullaut-Valera, ArbaizaDiaz, Arrúe-Ruilobal, Coullaut-Valera \& Bajo-Bretón, 2011; Fernández-Serrano, Pérez-García \& Verdejo-García, 2011; Jacobus \& Tapert, 2013; Madoz-Gúrpide \& OchoaMangado, 2012; Mena et al, 2013; Mota \& Corral, 2011; Pagani, 2014; Rosselli \& Ardila, 1996).

Específicamente, dentro de las áreas cerebrales implicadas en la atención se destaca la participación de los circuitos frontales derechos en tareas de atención sostenida, al igual que el córtex parietal posterior derecho, que está relacionado con la atención selectiva y sostenida (Cuervo \& Quijano, 2008); y tras áreas asociadas, como lo son el circuito corticostriatal (la circunvolución cingulada anterior, la amígdala y el núcleo accumbens), en el cual se produce un aumento de dopamina como consecuencia del consumo de droga y una predisposición para que el sujeto fije su atención en la misma (Franken, 2003). De este modo, por sus afectaciones fisiológicas en el cerebro, aquellos estímulos que quedan asociados con el consumo de droga ayudan a provocar y mantener la búsqueda de estas mismas sustancias (Belin, Belin-Rauscent, Murray \& Everitt, 2013).

Ahora bien, para realizar un registro de ondas cerebrales y evaluar la forma en que las sustancias psicoactivas afectan el cerebro, por lo general se lleva a cabo un electroencefalograma (EEG), definido como: (a) una técnica de registro extracelular que mide la densidad de corriente de potenciales corticales (Silva, 2011), y (b) una prueba funcional cerebral que permite determinar la actividad eléctrica cortical a partir de un registro gráfico de la misma (Talamillo, 2011). En este procedimiento-conformado por una serie de electrodos cuya ubicación cerebral depende del Sistema Internacional 10-20 (González, Ortiz, Gutiérrez \& González, 2011)-, se representa la actividad cortical por medio de ondas cerebrales que tienen un bajo voltaje, por lo que se requiere de un amplificador para poder observarlas e interpretarlas (Silva, 2011).

En particular, las ondas cerebrales asociadas al proceso de atención en sujetos sanos son las ondas theta- $\theta$ de $4-7 \mathrm{~Hz}$, relacionadas con el apoyo que suministran a los procesos cognitivos, entre ellos, el aprendizaje (Lee, 2013), y las ondas alfa- $\alpha$ de 7-13 Hz (Carretie, 2009; Salgado, 2003), que se identifican en estados de alerta relajado (Lomas, Ivtzan \& Cynthia, 2015); sin embargo, con respecto al déficit de atención, algunos estudios han documentado la presencia de ondas theta- $\theta$ de $4-7 \mathrm{~Hz}$ y beta- $\beta$ de $13-30 \mathrm{~Hz}$, sobre todo 
en EEG realizados a sujetos con Déficit de Atención con Hiperactividad (TDAH) (Lansbergen, Arns, van DongenBoomsma, Spronk \& Buitelaar, 2011; van Hell et al., 2010).

Asimismo, diversos estudios han manifestado la presencia de diferencias en la potencia espectral de ondas tras el consumo de sustancias psicoactivas. En el caso del consumo de alcohol se ha identificado la presencia de ondas delta- $\delta$ de $0-4 \mathrm{~Hz}$ y beta- $\beta$ rápida de $10-35 \mathrm{~Hz}$ (Courtney \& Polich, 2010), así como una reducción de alfa- $\alpha(7-13 \mathrm{~Hz})$ (Ehlers \& Phillips, 2007), y un incremento de bandas theta- $\theta$ $(4-7 \mathrm{~Hz})$ y delta- $\delta(0-4 \mathrm{~Hz})$, cuyo incremento se asocia con atrofia cortical, lo que sugiere la posible existencia de una disfunción frontal y explica el menor rendimiento en pruebas psicológicas de atención (Camelo, Rojas, Mejía \& Castro, 2015; Quesada, Diaz-Pérez, Herrera, Tamayo-Porras \& Rubio-López, 2007) y el aumento de bandas rápidas, que ayuda a predecir la recaída al consumo (Bauer, 2001).

También, con respecto a estudios de EEG con cocaína o benzoilmetilecgonina, se ha identificado un incremento de beta- $\beta(13-30 \mathrm{~Hz})$ en regiones corticales centrales-frontales, así como un aumento de alfa- $\alpha(7-13 \mathrm{~Hz})$ en regiones frontales-temporales, siendo beta- $\beta$ mayor que alfa- $\alpha$ (Heming, Glover, Koeppl, Phillips \& London, 1994); un aumento de theta- $\theta(4-7 \mathrm{~Hz})$ y beta- $\beta(13-30 \mathrm{~Hz})$ en la corteza prefrontal (FP1-FP2) (Reid, Flammino, Howard, Nilsen \& Prichep, 2006); una disminución de theta- $\theta$ (4-7 Hz), beta- $\beta 1$ y beta- $\beta 2$ en regiones posteriores cerebrales y una tendencia de disminución de delta- $\delta$ en regiones parietales (Copersino, Heming, Better, Cadet \& Gorelick, 2009); una disminución de alfa- $\alpha(7-13 \mathrm{~Hz})$, un aumento tónico de theta- $\theta(4-7 \mathrm{~Hz})$ y una disminución tónica de delta- $\delta(0-4 \mathrm{~Hz})$ (Kiyatkin \& Smimov, 2010); una reducción de alfa- $\alpha(7-13 \mathrm{~Hz})$ tras la exposición a estímulos asociados al consumo de cocaína (Liu, Vaupel, Grant \& London, 1998); y un desequilibrio hemisférico izquierdo producido por el aumento de delta- $\delta$ (de 0-4 Hz) ante respuestas a condiciones gratificantes en sujetos dependientes (Balconi \& Finocchiaro, 2015). De igual forma, el EEG en individuos que han consumido cannabis y se encuentran en un periodo de abstinencia se caracteriza por la presencia de ondas beta y gamma (Allsop \& Copeland, 2016).

Por otra parte, investigaciones de EEG en consumidores de éxtasis o 3.4 Metilendioximetanfetamina, como la de Adamaszek, Khaw, Buck, Andresen y Thomasius (2010), mostraron que los consumidores de esta sustancia presentan: (a) un aumento de beta- $\beta$ (13-30 Hz) en comparación con los controles; (b) una baja actividad de alfa- $\alpha(7-13 \mathrm{~Hz})$ y theta- $\theta$ (4-7 Hz); y (c) un aumento de theta- $\theta(4-7 \mathrm{~Hz})$ en usuarios de medio y alto consumo.

Igualmente, con respecto a los estudios de EEG en sujetos consumidores de nicotina, se ha encontrado un aumento de beta- $\beta$ (13-30 Hz) sobre el lóbulo frontal izquierdo y posterior en fumadores expuestos a estímulos relacionados con el hecho de fumar (Littel, Franken \& van Strien, 2009) y un aumento significativo de frecuencia alfa- $\alpha$ (7$13 \mathrm{~Hz}$ ) ante estímulos como el cigarrillo (Cui et al., 2013). Asimismo, investigaciones de EEG en sujetos consumidores de opioides han expresado un aumento de alfa- $\alpha(7-13 \mathrm{~Hz})$ y una disminución de delta- $\delta(0-4 \mathrm{~Hz})$ (Phillips, Heming \& London, 1994); mientras que en consumidores de heroína se ha encontrado un aumento significativo de delta- $\delta(0-7 \mathrm{~Hz})$ (Greenwald \& Roehrs, 2005). Existen otros estudios cuyo objetivo ha sido el estudio de cambios en el EEG en sujetos consumidores de distintas sustancias psicoactivas (Ceballos, Tivis, Prather \& Nixon, 2008; Lansbergen, Dumont, Van Gerven, Buitelaar \& Verke, 2011).

Registro de la actividad eléctrica cerebral mediante BCI

El Brain Control Interface (BCI) se define como la tecnología que, por medio de la adquisición de ondas cerebrales, le permite al individuo actuar recíprocamente con su entorno (Santana, Ramírez \& Ostrosky-Solís, 2004). Con este método, la actividad cerebral se registra por medio de un EEG y posteriormente se procesa con el fin de distinguir tareas o estados mentales (Flórez, Azorín, Úbeda \& Fernández, 2011) producto de los cambios en la actividad neuronal (Zhao \& Zhang, 2007). Durante los últimos años, esta tecnología ha motivado a numerosos investigadores a desarrollar una interfaz cerebro-computador eficiente y confiable que sigue un mismo principio básico (registro de la actividad cerebral, procesamiento y caracterización de la señal, y la interacción con el entorno de acuerdo con los propósitos del usuario), independientemente de la forma en que se opera la tecnología de los dispositivos utilizados en los diferentes grupos de investigación.

La mayoría de estudios con tecnología BCI se han realizado con los objetivos de: (a) restablecer el movimiento de neuroprótesis en pacientes con tetraplejia por medio de la actividad eléctrica cerebral, la cual representa mensajes (Nicolas \& Gómez, 2012); (b) potenciar la comunicación en pacientes tetrapléjicos a partir de un tablero $6 \times 6$, lo cual se relaciona con el potencial evocado P300 que ha sido utilizado como un dispositivo que proporciona la comunicación (Duvinage, Castermans \& Dutoit, 2012); (c) obtener imágenes en movimiento según señales audiovisuales presentadas por un ordenador y un altavoz (Choi, Ryu, Lee \& Lee, 2011); (d) evaluar los artefactos EEG correspondientes al movimiento o el parpadeo de los ojos (Bobrov et al., 2011); (e) identificar el tipo de señales fisiológicas que registra el EPOC EMOTIV (Duvinage et al., 2012); (f) registrar el EEG con respecto a tareas mentales 
en sujetos sanos (Flórez et al., 2011); y (g) desarrollar y evaluar los comandos del BCI que pacientes tetrapléjicos podrían aprender a controlar (Kauhanen et al., 2007).

Sin embargo, son escasos los estudios que evidencian la aplicación de estos dispositivos en el estudio de los procesos cognoscitivos e, incluso, en las problemáticas como el consumo de sustancias psicoactivas; y es por esto que la presente investigación tiene como objetivo registrar la actividad eléctrica cerebral (EEG) en tareas de atención (sostenida y selectiva) en un grupo de adolescentes policonsumidores frente a un grupo cuasi-control, por medio de un instrumento Epoc Emotiv BCI (Brain Control Interface).

\section{Método}

Diseño

Se utilizó un diseño ex post facto retrospectivo con grupo cuasi control (León \& Montero, 2003). El grupo objetivo estuvo conformado por un conjunto de adolescentes con el mismo valor en la variable independiente presencia de policonsumo, mientras que el grupo cuasi-control se caracterizó por un grupo de adolescentes que no presentaba ese valor en dicha variable, aunque eran bastante similares a los del grupo objetivo en las variables que se querían controlar.

\section{Sujetos}

La muestra estuvo conformada por un grupo de 46 adolescentes varones ( 23 policonsumidores pertenecientes al Centro de Orientación Juvenil Luis Amigó y 23 cuasicontroles de un colegio oficial de Bogotá) con edades entre los 12-17 años ( $M=14.60 ; S D=1.67)$, seleccionados por medio de un muestreo no-probabilístico auto-selectivo, y con características sociodemográficas similares (nivel educativo: básico primaria; estrato socioeconómico: 1-2). Se tuvo en cuenta que los participantes no tuvieran antecedentes neurológicos, trastornos psiquiátricos, enfermedades orgánicas ni diagnóstico de déficit cognoscitivo. El grupo de policonsumidores registró una historia de policonsumo de mínimo un año, con un periodo de abstinencia de al menos un mes y un consumo mínimo de tres sustancias psicoactivas.

\section{Instrumentos}

La prueba de detención de consumo de alcohol, tabaco y sustancias (ASSIST)

Se utilizó la versión 3.1 del ASSIST (Organización Mundial de la Salud, 2011), una prueba dirigida a personas con consumo de sustancias psicoactivas, cuyo objetivo es el de informar el riesgo para cada sustancia que el usuario informa haber consumido. En particular, consta de ocho preguntas y un tiempo de ejecución de 5-10 minutos.

Emotiv EPOC research grade 14 Channel Mobile EEG (Emotiv Inc, 2011).

El EPOC Emotiv (Emotiv Inc, 2011) es un sistema que detecta y amplifica la señal eléctrica cerebral a partir de un casco que contiene 14 electrodos superficiales (AF3, F7, F3, FC5, T7, P7, O1, O2, P8, T8, FC6, F4, F8, AF4) y dos de referencia ( $\mathrm{P} 3$ y P4), basados en el sistema internacional 10-20. Específicamente, el instrumento utiliza el método de muestreo secuencial -solo ADC (conversione analogicodigitale)- a una velocidad de 128 o 256 SPS $(2.048 \mathrm{~Hz}$ interno), y a una resolución de 14 bits; el ancho de banda se encuentra entre $0.2-43 \mathrm{~Hz}$, con filtros notch digitales a $50 \mathrm{~Hz}$ y $60 \mathrm{~Hz}$; el filtrado es construido en forma digital sincrónica y de quinto orden; el rango dinámico (entrada de referencia) es de $8400 \mu \mathrm{V}$ pico a pico; el modo de acople es AC (current alternative); y la conectividad es wireless banda $2.4 \mathrm{G} \mathrm{Hz}$.

Programa virtual de entrenamiento cerebral "Brain HQ", módulo "enfoco mi atención" (Merzenich, 2003).

Este módulo del programa de entrenamiento cerebral Brain HQ permite medir y evaluar la atención sostenida y selectiva, además de obtener el tiempo de respuesta de cada participante. Específicamente, se trata de la presentación de una serie de parejas de figuras geométricas (estímulos visuales) de diferentes colores y formas a través de un ordenador, por medio de dos tareas: (a) la selección por color, que consiste en que el sujeto oprima la tecla "SI" cada vez que ambas figuras geométricas tengan el mismo color y oprima "NO" cada vez que ambas figuras tengan un color diferente; y (b) la selección por forma, que consiste en que el participante oprima la tecla "SÍ" cada vez que ambas figuras geométricas tengan la misma forma y oprima "NO" cada vez que ambas figuras tengan una forma diferente.

El tiempo de ejecución de cada tarea corresponde a dos a tres minutos, aproximadamente, dependiendo de la ejecución de cada participante. Ambas tareas implican procesos de atención sostenida y selectiva bajo un paradigma Go no $G o$, pero se diferencian en el tipo de información (color o forma) que el sujeto debe discriminar.

\section{Procedimiento}

Una vez seleccionada la muestra, esta se organizó por grupos de edad (12, 13, 14, 15, 16 y 17 años) y se asignaron ocho adolescentes a cada grupo objetivo y cuasi-control -sin 
180

embargo, para el grupo de 12 años solo se incluyeron seis adolescentes-. Tras haber asignado los grupos, se prosiguió con una primera fase de creación de historia clínica y la aplicación del ASSIST; posteriormente, una segunda fase, de preparación, que consistió en brindar instrucciones y recomendaciones al participante para ser aplicadas durante el registro de la actividad eléctrica cerebral durante las tareas; $\mathrm{y}$, por último, y consecutivamente con la anterior, una tercera fase en la que se llevó a cabo el Registro Eléctrico Cerebral mediante el software Emotiv Xavier Test Bench en un ambiente controlado, al colocar al sujeto en frente del ordenador e iniciar la captación de la actividad eléctrica cerebral correspondiente a los canales AF3, AF4, F3, F4, F7, F8, FC5, FC8 para ambas tareas (A y B) del programa virtual de entrenamiento cerebral "Brain HQ", módulo "enfoco mi atención".

\section{Análisis de datos}

Se utilizó un análisis de frecuencia para los datos correspondientes al ASSIST que permitió obtener el número de sujetos consumidores por cada una de las sustancias psicoactivas y la frecuencia de consumo por cada una. Para determinar el tipo de distribución de la variable Tiempos de respuesta del programa virtual de entrenamiento cerebral "Brain HQ", módulo "enfoco mi atención", se utilizó la prueba de Kolmogorov-Smirnov. Esta prueba arrojó una distribución no normal $(\mathrm{p}<.05)$, por lo que se aplicó el test de U de Mann-Whitney, correspondiente a una prueba no paramétrica. Los análisis estadísticos se realizaron mediante el software SPSS, versión 20.

Con respecto al análisis de registros eléctricos cerebrales, obtenidos con el software Emotiv Xaier Test Bench, se llevó a cabo la lectura y descripción de cada uno de ellos, donde se identificaron los grafo-elementos asociados con la atención selectiva y sostenida en ambos grupos para ambas tareas.

Y el análisis cuantitativo de los registros eléctricos cerebrales se realizó mediante la Escala de Transformación de Fourier - con filtros de tipo pasabanda que usan la función firpmode del software MATLAB-a partir del cual se tuvieron en cuenta las frecuencias de $3-4 \mathrm{~Hz}$ para delta- $\delta$, 4-7 Hz para theta- $\theta, 7-13 \mathrm{~Hz}$ para alfa- $\alpha$ y $13-30 \mathrm{~Hz}$ para beta- $\beta$. El proceso de filtraje se comenzó a partir de $3 \mathrm{~Hz}$, ya que era probable que en las frecuencias entre $0-2 \mathrm{~Hz}$ y mayores a $45 \mathrm{~Hz}$ hubiera presencia de ruido. Además, el EPOC Emotiv dentro de sus características presenta un filtro pasabanda de $0.2-45 \mathrm{~Hz}$ con el fin de controlar efectos de impedancia. Asimismo, se realizó un proceso de segmentación para cada uno de los registros, donde se procesaron segmentos de tan solo 70-90 segundos-tiempo asociado a las tareas de atención con el fin de obtener la señal relacionada con las mismas-. Lo anterior permitió obtener gráficas de densidad espectral de potencia para cada uno de los participantes con respecto a cada uno de los canales y tareas de atención seleccionados.

\section{Consideraciones éticas}

Para este estudio se tuvo en cuenta las consideraciones expuestas en el Código Deontológico y Ético del Psicólogo (Colegio Colombiano de Psicólogos, 2013), tales como: (a) la confidencialidad, mediante la cual se establece que el psicólogo no podrá revelar aquella información confidencial y privada del paciente, a menos que sea bajo su consentimiento o del representante legal de la persona (ley 1090 del 2006, título II, articulo 2, numeral 5); (b) el secreto, el cual establece que se debe mantener la confidencialidad, confiabilidad y credibilidad en el cumplimiento de los compromisos (ley 1164 del 2007, artículo 36); y (c) el secreto profesional, mediante el cual se establece que el secreto profesional es inviolable (Constitución Nacional de 1991, artículo 74).

\section{Resultados}

A continuación se muestra el análisis de frecuencia de los datos correspondientes al ASSIST, el análisis cuantitativo de los tiempos de respuesta del programa virtual de entrenamiento cerebral "Brain HQ", módulo "enfoco mi atención", y el análisis cuantitativo de los registros eléctricos cerebrales mediante la Escala de Transformación de Fourier, soportado por algunos de los registros electroencefalográficos.

\section{Prueba de detención de consumo de alcohol, tabaco y sustancias (ASSIST)}

La prueba de detención de consumo permitió identificar las sustancias consumidas por parte del grupo policonsumidor. Dentro de este grupo, el $95.7 \%$ de los participantes afirmó haber consumido tabaco; el $100 \%$, alcohol; el $100 \%$, cannabis; el $95.7 \%$, inhalantes; y el $87 \%$, sedantes (véase Figura 1).

De igual forma, con respecto a la frecuencia de consumo para cada una de las sustancias por parte del grupo policonsumidor, la mayor parte de los sujetos afirmaron haber consumido diariamente tabaco (65\%), cannabis $(78.3 \%)$ e inhalantes (39.1\%); comparado con aquellos que manifestaron haber consumido semanalmente alcohol $(78.3 \%)$ y sedantes (34.8 \%) (véase Figura 2$)$. 


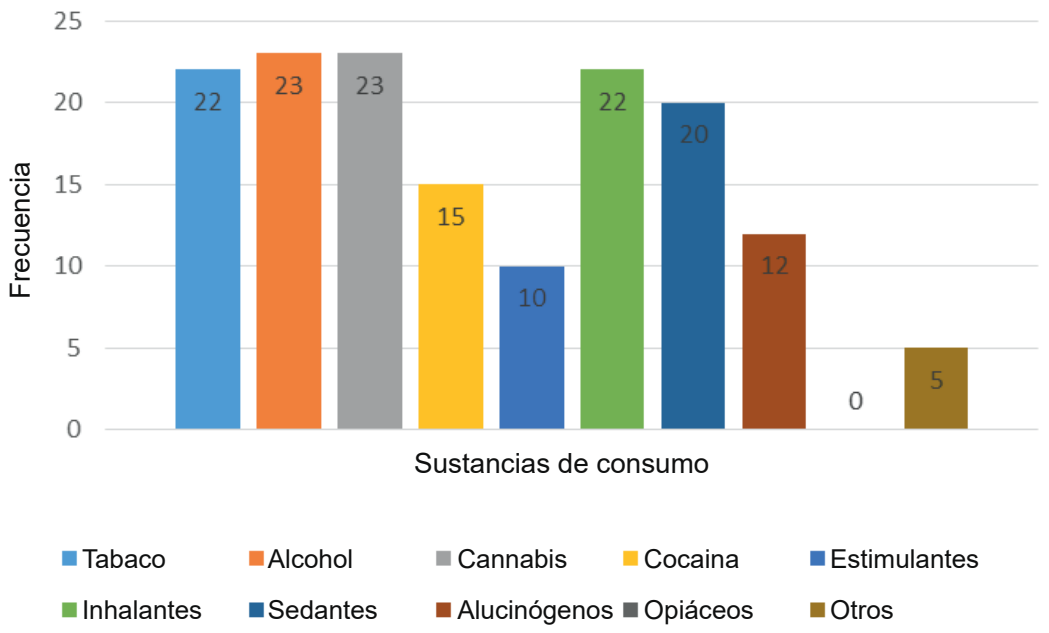

Figura 1. Sustancias consumidas en el grupo objetivo.

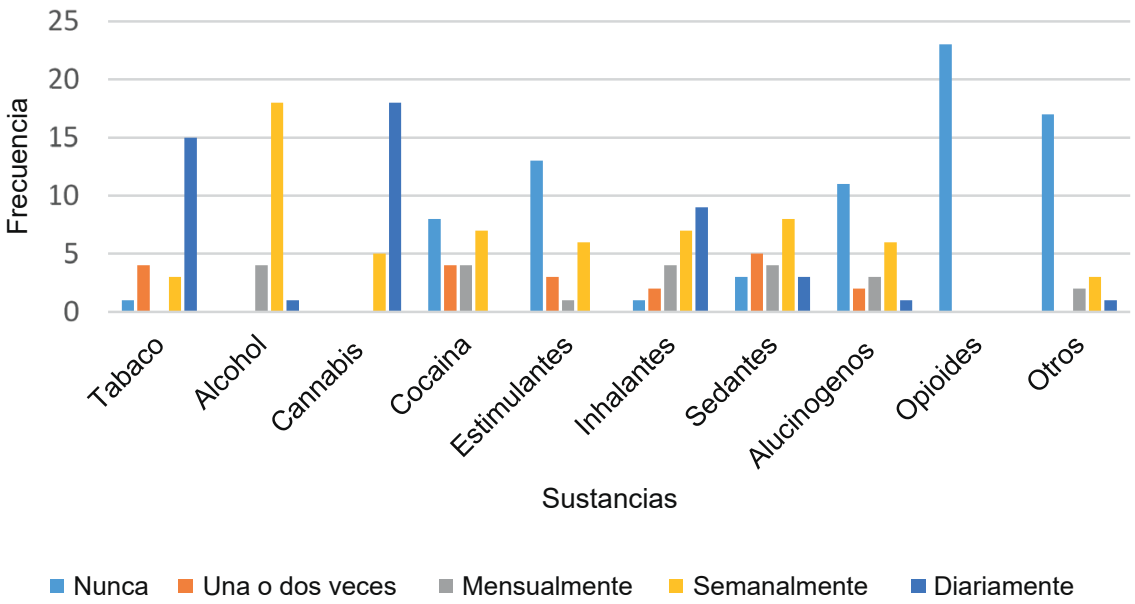

Figura 2. Frecuencia del consumo de sustancias en el grupo objetivo.

Tiempos de respuesta en el programa virtual de entrenamiento cerebral "Brain HQ", módulo "enfoco mi atención"

Se utilizó un estadístico no paramétrico de la U de Mann-Whitney para identificar diferencias entre el grupo de adolescentes policonsumidores y los sujetos cuasi-controles con respecto a los tiempos de respuesta para ambas pruebas de atención (A y B) (véase Tabla 1).

Tabla 1.

Test de U de Mann-Whitney para tiempos de respuesta por grupo

\begin{tabular}{cccc}
\hline Grupo/Prueba & & A & B \\
\hline Objetivo & \multirow{2}{*}{ Mediana } & 31.07 & 31.91 \\
Cuasi-control & & 15.93 & 15.09 \\
Z & & -3.840 & -4.263 \\
Significancia & & $* * .000$ & $* * .000$ \\
\hline
\end{tabular}

Nota $. \mathrm{A}=$ tarea de selección de color; $\mathrm{B}=$ tarea de selección de forma. ** Significancia menor a .01 .
Una vez finalizado el test de U de Mann-Whitney, se identificó una diferencia significativa al .01 en el promedio de rango para cada grupo con respecto a cada tarea de atención, lo cual quiere decir que los adolescentes policonsumidores obtuvieron una mediana mayor en ambas tareas con respecto a los adolescentes cuasi-controles, y, por tanto, se puede afirmar que el grupo de consumidores tuvo mayores tiempos de respuesta en cada una de las tareas con respecto al grupo cuasi-control (véase Tabla 1).

Registro de la actividad cerebral durante la prueba de atención

Tras el análisis tanto cualitativos -que permitieron identificar los grafo-elementos- y cuantitativos -a partir de la Escala de Transformación de Fourier de los registros EEG, que permitió obtener graficas de densidad espectral de potencia-, se observó que durante la ejecución del ejercicio "enfoco mi atención", en el grupo de policonsumidores 
hubo una predominancia de ondas combinadas theta- $\theta$ (4-7 Hz) - beta- $\beta(13-30 \mathrm{~Hz})$ (véase Figura 3$)$ y theta- $\theta$ (4-7 Hz) - delta- $\delta(3-4 \mathrm{~Hz}$ ) (véase Figura 5) en los canales AF3, AF4, F3, F4, F7, F8, FC5 y FC8, correspondientes a las áreas cerebrales prefrontales y frontales durante las tareas A y B para la mayoría de participantes; mientras que en el grupo cuasi-control de adolescentes hubo una prevalencia de ondas combinadas theta (4-7 Hz) - alfa- $\alpha$ (7-13 Hz) en los canales AF3, AF4, F3, F4, F7, F8, FC5 y FC8 durante la ejecución de ambas tareas A y B para la mayoría de los participantes de los diferentes grupos de edad (véase Figuras 4 y 6 ).

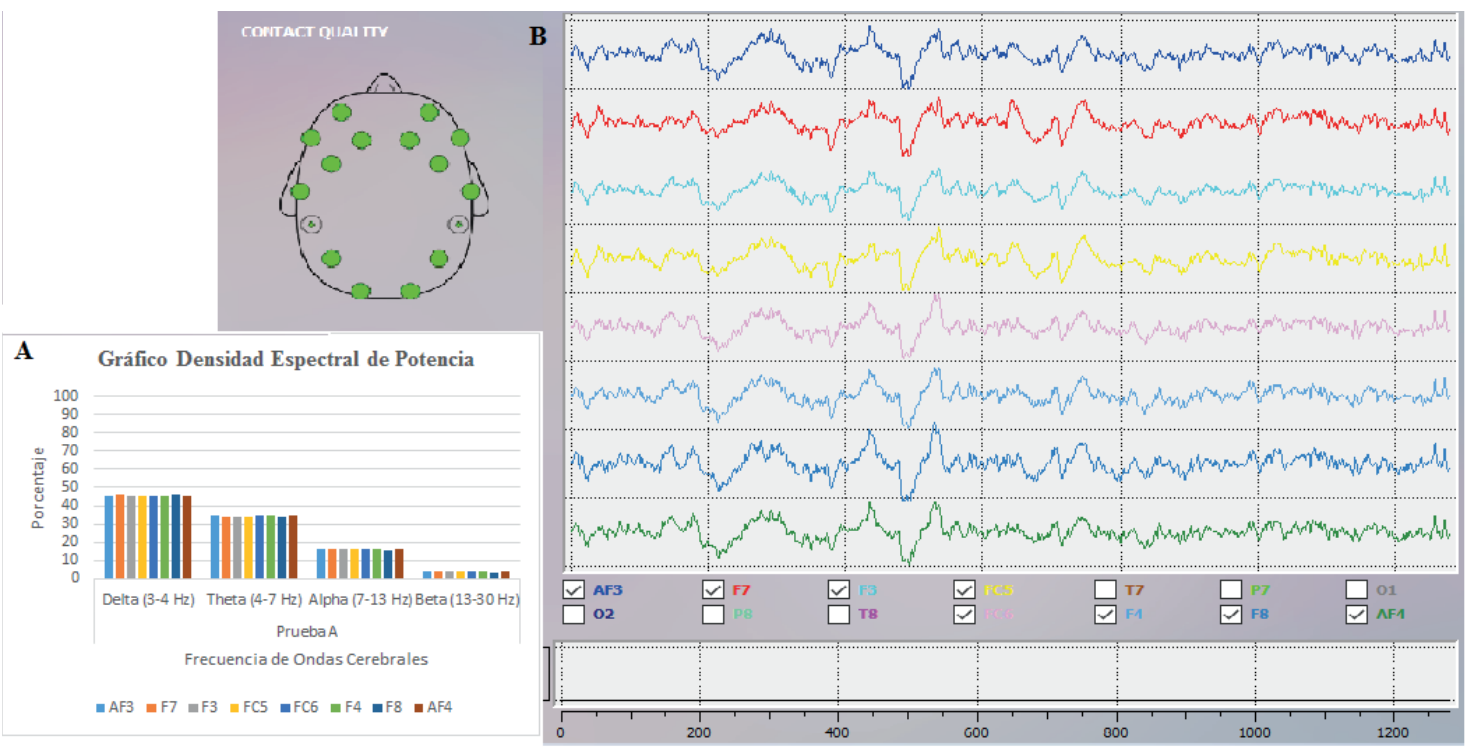

Figura 3. A) Densidad Espectral de Potencia y B) Registro EEG con el Test de Bench durante la tarea A de atención selectiva y sostenida del Programa virtual de entrenamiento "Brain HQ", módulo "enfoco mi Atención", en un adolescente policonsumidor. La gráfica y registro EEG asociados a los canales AF3, AF4, F3, F4, F7, F8, FC5 y FC6 muestran una predominancia de ondas theta- $\theta$ - delta- $\delta$.
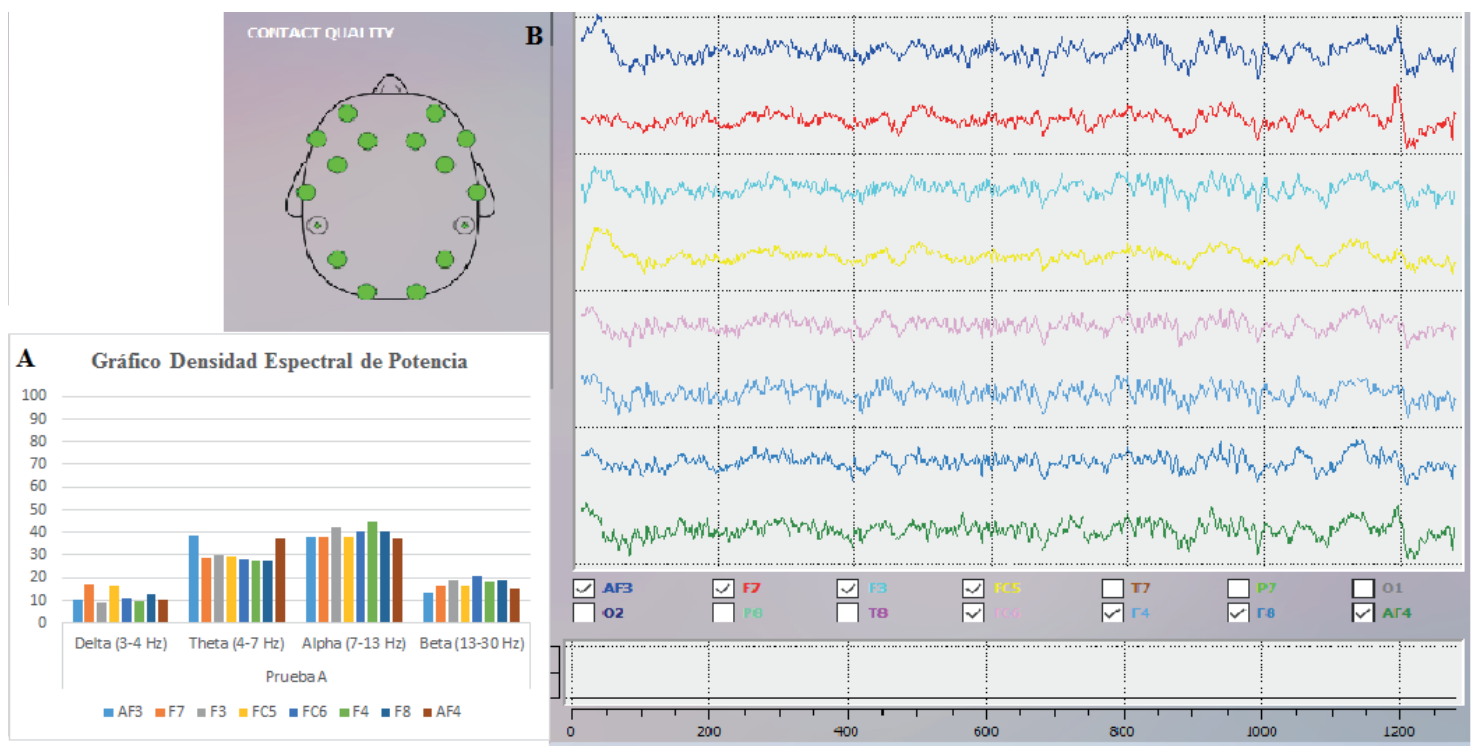

Figura 4. A) Densidad Espectral de Potencia y B) Registro EEG con el Test de Bench durante la tarea A de atención selectiva y sostenida del Programa virtual de entrenamiento "Brain HQ", módulo "enfoco mi atención", en un adolescente del grupo cuasi-control. La gráfica y registro EEG asociados a los canales AF3, AF4, F3, F4, F7, F8, FC5 y FC6 muestran una predominancia de ondas theta- $\theta$ - alfa- $\alpha$. 

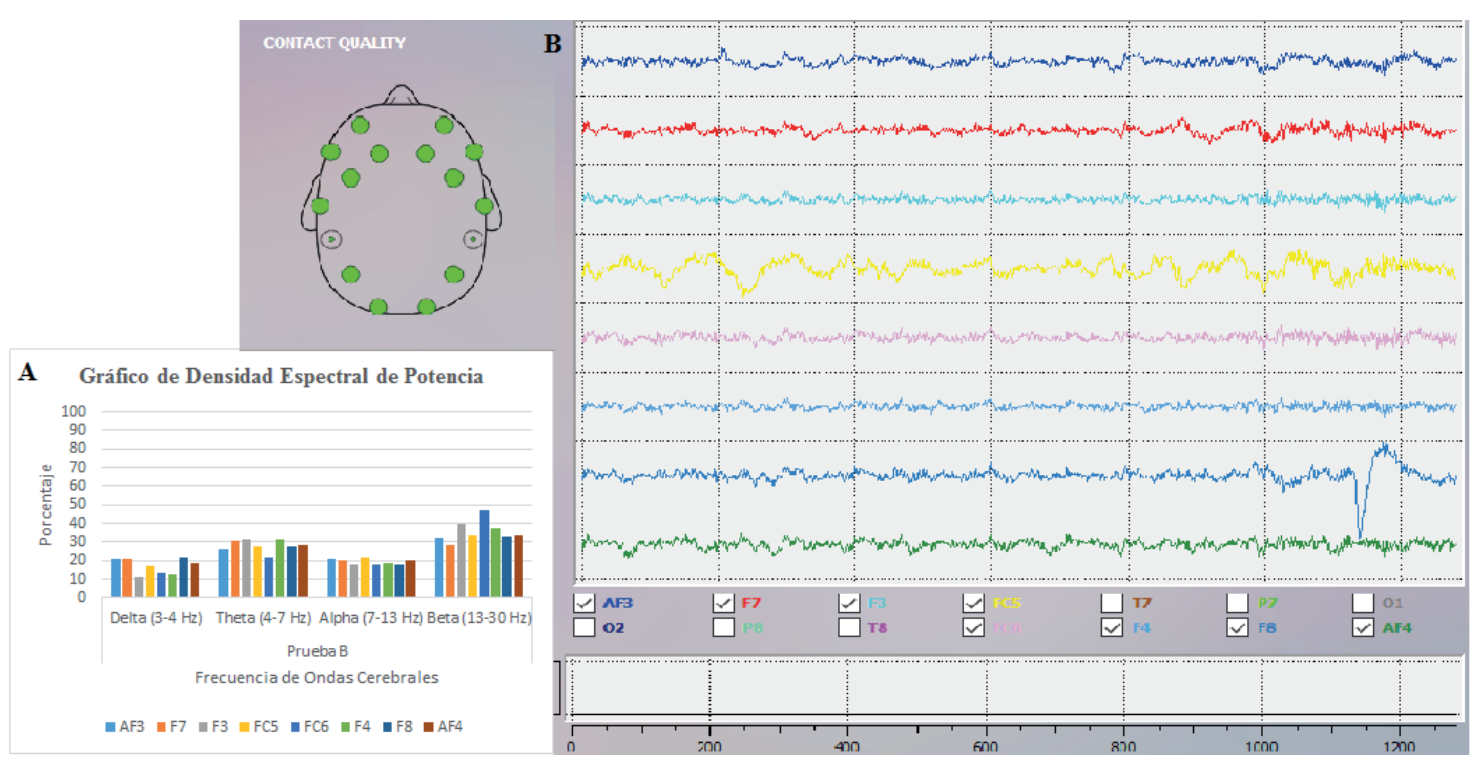

Figura 5. A) Densidad Espectral de Potencia y B) Registro EEG con el Test de Bench durante la tarea B de atención selectiva y sostenida del Programa virtual de entrenamiento "Brain HQ", módulo "enfoco mi atención", en un adolescente policonsumidor. La gráfica y el registro EEG asociados a los canales AF3, AF4, F3, F4, F7, F8, FC5 y FC6 muestran una predominancia de ondas theta- $\theta$ - beta- $\beta$.
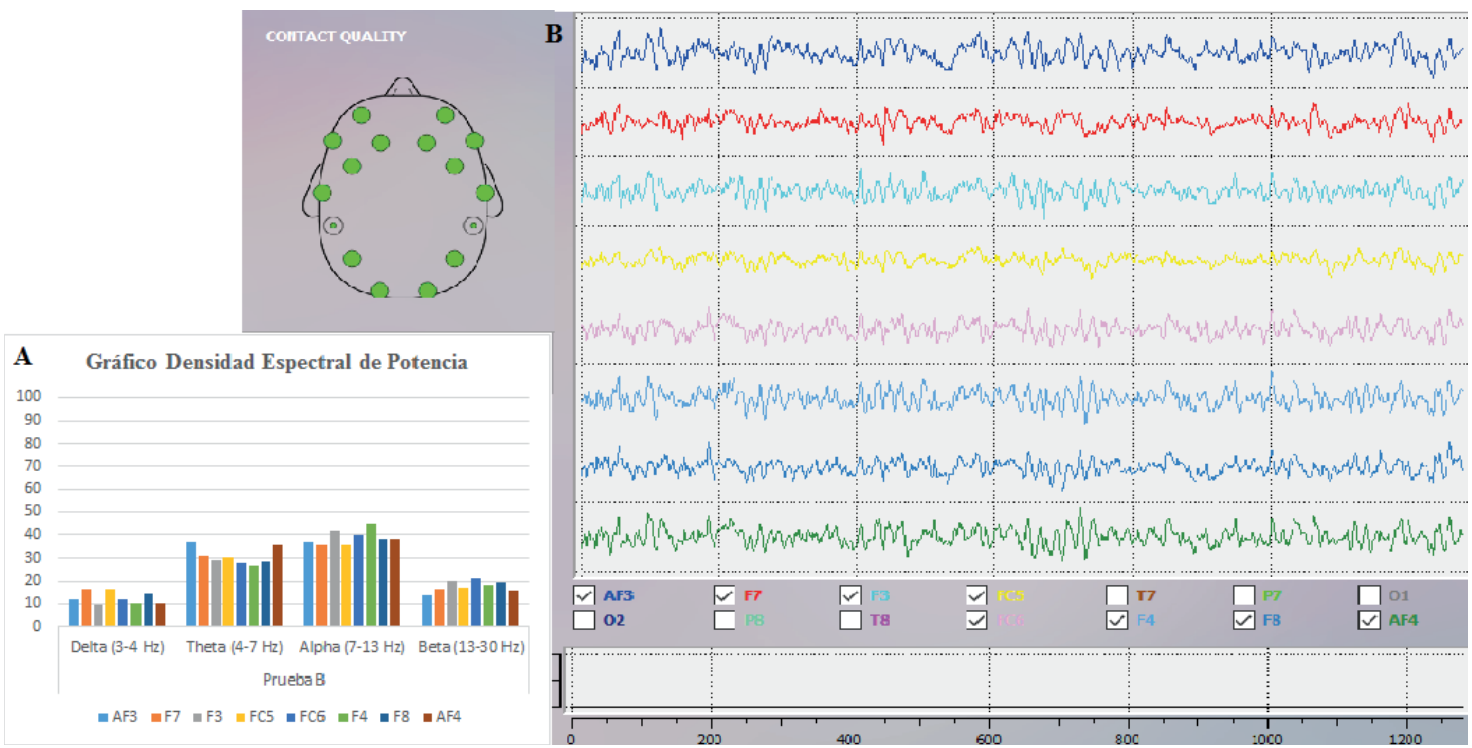

Figura 6. A) Densidad Espectral de Potencia y B) Registro EEG con el Test de Bench durante la tarea B de atención selectiva y sostenida del Programa virtual de entrenamiento "Brain HQ", módulo "enfoco mi atención" en un adolescente del grupo cuasi-control. La gráfica y el registro EEG asociados a los canales AF3, AF4, F3, F4, F7, F8, FC5 y FC6 muestran una predominancia de ondas theta- $\theta$ - alfa- $\alpha$.

\section{Discusión}

La presente investigación tuvo el objetivo de registrar la actividad eléctrica cerebral (EEG) durante tareas de atención (sostenida y selectiva) en adolescentes policonsumidores por medio de un instrumento Epoc Emotiv BCI (Brain Control Interface). Los resultados obtenidos muestran la presencia de ondas theta- $\theta(4-7 \mathrm{~Hz})$ - beta- $\beta(13-30 \mathrm{~Hz})$ y theta- $\theta(4-7 \mathrm{~Hz})$ - delta- $\delta(3-4 \mathrm{~Hz})$ en el grupo de adolescentes policonsumidores en áreas prefrontales y frontales 
asociadas con los canales AF3, AF4, F3, F4, F7, F8, FC5 y FC6, y estas, a su vez, con el proceso atencional (Squire, Noudoost, Schafer \& Moore, 2013).

La presencia de ondas theta- $\theta(4-7 \mathrm{~Hz})$ - beta- $\beta$ (13$30 \mathrm{~Hz}$ ) en adolescentes policonsumidores es un hallazgo que se correlaciona con registros electroencefalográficos de sujetos con TDAH que presentan un patrón electrofisiológico similar; lo cual indica la posible existencia de alteraciones en el proceso atencional de adolescentes policonsumidores (Adamaszek et al., 2010; Heming et al., 1994; Kiyatkin \& Smimov, 2010; Lansbergen et al., 2011; Reid et al., 2006; Van Hell et al., 2011). Esto es compatible con el hecho de que el policonsumo es un trastorno que se caracteriza por la desinhibición conductual (Lacono et al. 2008) y la disminución en la capacidad para regular el control sobre el deseo (Feil et al., 2010), lo cual, a su vez, es algo característico en sujetos con TDAH.

Asimismo, el aumento de ondas theta- $\theta$ (4-7 Hz) delta- $\delta(3-4 \mathrm{~Hz})$ identificado en el presente estudio en adolescentes policonsumidores sugiere una hipoactividad en zonas frontales y prefrontales asociadas a problemas como el enlentecimiento atencional, la atrofia cortical, la disfunción frontal y un bajo rendimiento en tareas de atención; resultados equiparables a los encontrados en los estudios realizados por Adamaszek et al. (2010); Balconi y Finocchiaro (2015); Courtney y Polich (2010); Greenwald y Roehrs (2005); y Quesada et al. (2007).

Con respecto a los adolescentes cuasi-controles, en este trabajo se identificó una predominancia de ondas theta- $\theta$ (4-7 Hz) - alfa- $\alpha(7-13 \mathrm{~Hz})$ en áreas prefrontales y frontales asociadas a los canales AF3, AF4, F3, F4, F7, F8, FC5 y FC6 en la mayoría de la muestra, sobre todo en los grupos de 12, 14,15 y 16 años de edad durante las tareas de atención A y B. Específicamente, estas ondas son representativas del proceso atencional, ya que distintas fuentes afirman que la presencia de ondas theta- $\theta(4-7 \mathrm{~Hz})-$ alfa- $\alpha(7-13 \mathrm{~Hz})$ se relacionan con dicho proceso (Lee, 2013; Lomas et al., 2015). Y aunque las ondas theta- $\theta(4-7 \mathrm{~Hz})$ se observan cuando se produce una disminución en el nivel de activación (Nicolas \& Gómez, 2012), estas mismas también están presentes y asociadas con estados que requieren de mucha concentración durante la realización de tareas que implican el proceso atencional y la memoria de trabajo (Carretie, 2009; Salgado, 2003).

De acuerdo con lo anterior, estos resultados sugieren que el consumo de sustancias psicoactivas genera cambios en la actividad eléctrica cerebral de los adolescentes, y que dicha actividad se refleja en los déficits atencionales observados en adolescentes policonsumidores $y$, por tanto, constituye un posible factor de riesgo para el inicio y mantenimiento del consumo de sustancias.
Por otra parte, con respecto a los tiempos de respuesta, estos fueron mayores en los adolescentes consumidores en comparación con los adolescentes del grupo cuasi-control durante la ejecución de ambas tareas de atención Ay $\mathrm{B}$ (véase Tabla 1), y este hallazgo se podría asociar a la presencia de una alteración o enlentecimiento en el procesamiento de información visual en participantes policonsumidores, que afecta la ejecución en tareas de atención sostenida y selectiva (Acosta et al., 2009; Fernández-Serrano et al., 2011; Jacobus \& Tapert, 2013; Mota \& Corral, 2011).

Finalmente, esta investigación aporta datos importantes al campo de la psicofisiología de la actividad eléctrica cerebral que permiten y ayudan a caracterizar posibles perfiles electrofisiológicos asociados a la población adolescente consumidora de sustancias psicoactivas, además de que podrían tenerse en cuenta a la hora de realizar la evaluación, diseño y planeación de nuevas estrategias de intervención, como la retroalimentación en este tipo población. Este trabajo constituye un primer esfuerzo por vincular las nuevas tecnologías de registro electrofisiológico cerebral al estudio de aspectos psicobiológicos del consumo de sustancias psicoactivas, pero una de las limitaciones de este estudio es el tamaño de la muestra, por lo que sería interesante que futuras investigaciones trabajen con muestras más grandes con el objetivo de contrastar o duplicar los resultados obtenidos en la presente investigación.

\section{Referencias}

Acosta, J., Cervantes, M. L., \& Puentes, P. (2009). Perfil del Mini-Mental en policonsumidores de 25-50 años del área metropolitana de la ciudad de Barranquilla-Colombia. Psicogente, 12(22), 316-325. doi: 10.17081/psico.12.22.1062

Acosta, J., Cervantes, M. L., Pineda, W. F., Torre, G., López, L., \& Cárdenas, B (2011). Policonsumo desde una perspectiva neuropsicológica. Psicogente, 14(25), 178-189. Recuperado de http://revistas.unisimon.edu.co/index.php/psicogen te/article/view/1865/1781

Adamaszek, M., Khaw, A. V., Buck, U., Andresen, B., \& Thomasius, R. (2010). Evidence of Neurotoxicity of Ecstasy: Sustained Effects on Electroencephalographic Activity in Polydrug Users. PLoS ONE, 5(11), 1-6. doi:10.1371/jour nal.pone.0014097

Allsop, D. J., \& Copeland, J. (2016). Age at first cannabis use moderates EEG markers of recovery from cannabis. Journal of substance use, 21(4), 400-406. doi: 10.3109/14659891.2015.1040090

American Psychriatric Association (APA). (2005). Manual Diagnostico y Estadístico de los Trastornos Mentales DSM $I V$-TR (Edición española). España: Masson. 
Balconi, M., \& Finocchiaro, R. (2015). Decisional impairments in cocaine addiction, reward bias, and cortical oscillation "unbalance". Neuropsychiatric Disease and Treatment, 11, 777-786. doi: 10.2147/NDT.S79696

Bauer, L. O. (2001). Predicting Relapse to Alcohol and Drug Abuse via Quantitative Electroencephalography. Neuropsychopharmacology, 25(3), 332-340. doi: 10.1016/S0893133X(01)00236-6

Belin, D., Belin-Rauscent, A., Murray, J. E., \& Everitt, B. J. (2013). Addiction: failure of control over maladaptive incentive habits. Current opinion in neurobiology, 23(4), 564572. doi: 10.1016/j.conb.2013.01.025

Bobrov, P., Frolov, A., Charles, C., Fedulova, I., Bakhnyan, M., \& Zhavoronkov, A. (2011). Brain-Computer Interface Based on Generation of Visual Images. Open Access Freely Available Online, 6(6), 1-12. doi: 10.1371/journal. pone. 0020674

Caballero, L. (2005). Adicción a cocaína: Neurobiología, clínica, diagnóstico y tratamiento. Madrid: Ministerio de Sanidad y Consumo.

Camelo, S., Rojas, D., Mejía, A., \& Castro, R. (2015). Registro de la actividad eléctrica cerebral de la atención implicada en la conducción bajo el efecto del alcohol usando un instrumento BCI (Brain Control Interface). Revista Diversitas Perspectivas En Psicología, 11(2), 217-233. doi: 10.15332/ s1794-9998.2015.0002.04

Carretie, L. (2009). Psicofisiología. Madrid: Ed. Piramide.

Ceballos, N. A., Tivis, R., Prather, R., \& Nixon, S. J. (2008). Transdermal Nicotine Administration and the Electroencephalographic Activity of Substance Abusers in Treatment. NIH Public Access, 2(4), 202-214. doi: 10.1097/ ADM.0b013e31818b4e27

Choi, D., Ryu, Y., Lee, Y., \& Lee, M. (2011). Performance evaluation of a motor-imagerybased EEG-Brain computer interface using a combined cue with heterogeneous training data in BCI-Naive subjects. BioMedical Engineering OnLine, 10(91), 2-12. doi: 10.1186/1475-925X-10-91

Colado, M. I. (2008). Éxtasis (MDMA): estudios neurobiológicos en el laboratorio. Trastornos adictivos, 10(3), 183-189. doi: 10.1016/S1575-0973(08)76364-5

Colegio Colombiano de Psicólogos (2013). Deontología y bioética del ejercicio de la psicología en Colombia. Bogotá: El Manual Moderno S.A.

Colzato, L. S., Van den Wildenberg, W. P., \& Hommel, B. (2009). Reduced Attentional Scope in Cocaine Polydrug Users. PLOS ONE, 4(6), 1-5. doi: 10.1371/journal. pone. 0006043

Connolly, C. G., Foxe, J. J., Nierenberg, J., Shpaner, M., \& Garavan, H. (2012). The neurobiology of cognitive control in successful cocaine abstinence. Drug Alcohol Depend, 121(1-2), 45-53. doi: 10.1016/j.drugalcdep.2011.08.007

Copersino, M. L., Heming, R. I., Better, W., Cadet, J. L., \& Gorelick, D. A. (2009). EEG and cerebral blood flow velocity abnormalities in chronic cocaine users. Clinical EEG and Neuroscience, 40(1), 39-41. doi: 10.1177/155005940904000111

Corominas, M., Roncero, C., Bruguera, E., \& Casas, M. (2007). Sistema dopaminérgico y adicciones. Revista de neurología, 44(1), 23-31. Recuperado de https://dialnet.unirioja.es/ servlet/articulo? codigo $=2224138$

Coullaut-Valera, R., Arbaiza-Diaz del Rio, I., Arrúe-Ruilobal, R., Coullaut-Valera, J., \& Bajo- Bretón, R. (2011). Deterioro cognitivo asociado al consumo de diferentes sustancias psicoactivas. Actas Españolas de Psiquiatría, 39(3), 168173. Recuperado de https://www.actaspsiquiatria.es/reposi torio/13/71/ESP/13-71-ESP-168-173-776266.pdf

Courtney, K. E., \& Polich, J. (2010). Binge drinking effects on EEG in young adult humans. International Journal of Environmental Research and Public Health, 7(5), 2325-2336. doi:10.3390/ijerph7052325

Crespo-Fernández, J. A., \& Rodríguez, C. A. (2007). Bases neuroanatomicas, neurobiológicas y del aprendizaje de la conducta de adicción a la cocaína. Revista Latinoamericana de Psicología, 39(1), 83-107. doi: 10.14349/rlp.v39i1.572

Cuervo, M. T., \& Quijano, M. C. (2008). Alteraciones de la atención y su rehabilitación en trauma craneoencefálico. Pensamiento psicológico, 4(11), 167-182.

Cui, Y., Versace, F., Engelmann, J. M., Minnix, J. A., Robinson ... Cinciripini, P. M. (2013). Alpha Oscillations in response to affective and Cigarette-related stimuli in smoker. Nicotine y Tobacco research, 15(5), 917-924. doi: 10.1093/ntr/ nts 209

Daza, M. (2009). Efectos conductuales y neuroquímicos del consumo de éxtasis y cocaina en ratones adolescentes (Tesis doctoral). Departamento de Psicobiología, Universidad de Valencia, Valencia. Recuperado de http://www.tdx.cat/ handle $/ 10803 / 10203$ ? show $=$ full

D'Souza, M. S., \& Markou, A. (2011). Neuronal Mechanisms Underlying Development of Nicotine Dependence: Implications for Novel Smoking-Cessation Treatments. Addiction Science and Clinical Practice, 6(1), 4-17. Recuperado de https://www.ncbi.nlm.nih.gov/pmc/articles/PMC3188825/ pdf/ascp-06-1-4.pdf

Duvinage, M., Castermans, T., \& Dutoit, T. (2012). A P300BASED quantitative comparison between the emotiv epoc headset and a medical EEG device. LNMB Lab, 1-6. doi: 10.2316/P.2012.764-071

Ehlers, C. L., \& Phillips, E. (2007). Association of EEG alpha variants and alpha power with alcohol dependence in Mexican American Young Adults. Alcohol, 41(1), 13-20. doi: 10.1016/j.alcohol.2007.02.001

Emotiv inc (2011). Emotiv EPOC. Recuperado en: https://emo tiv.gitbook.io/epoc-user-manual/epoc+_headset_details/ universal_usb_receiver_dongle

Esel, E. (2006). Neurobiology of Alcohol Withdrawal: Inhibitory and Excitatory Neurotransmitters. Turkish Journal of Psychiatry, 17(2), 1-9. Recuperado de https://pdfs.seman 
ticscholar.org/aaa0/5a53fdc40f33a3d4ed182ca5050664e11 9f4.pdf

Fagundo, A. B., Martin-Santos, R., Abanades, S., Farré, M., \& Verdejo-García, A. (2008). Neuroimagen y adicción II: correlatos neuroantómicos y funcionales de la administración aguda, el craving y el consumo crónico de opiáceos, alcohol y cannabis. Revista Española de Drogodependencia, 32(2), 125-149. Recuperado de http://roderic.uv.es/bitstream/hand le/10550/22378/v33n2_1.pdf?sequence $=1 \&$ isAllowed $=y$

Feil, J., Sheppard, D., Fitzgerald, P. B., Yucel, M., Lubman, D. I., \& Bradshaw, J. L. (2010). Addiction, compulsive drug seeking, and the role of frontostriatal mechanisms in regulating inhibitory control. Neuroscience y Beobehavioral Reviews, 35(2), 248-275. doi: 10.1016/j.neubio rev.2010.03.001

Fernández-Espejo, E. (2006). Neurobiología de la adicción en psicoestimulante. Revista de Neurología, 43(3), 147-154. Recuperado de https:/idus.us.es/xmlui/bits tream/handle/11441/32261/neurobiologiadelaadiccion. pdf? sequence $=1 \&$ isAllowed $=\mathrm{y}$

Fernández-Serrano, M. J., Pérez-García, M., \& Verdejo-García, A. (2011).What are the specific Vs Generalized effects of drugs of abuse in neuropsychological performance. Neuroscience and Biobehavioral Reviews, 35(3), 377-406. doi: 10.1016/j.neubiorev.2010.04.008

Flórez, F., Azorín, J. M., Úbeda, A., \& Fernández, E. (2011). Development of a low-cost svm-based spontaneous braincomputer interfac. Biomedical Neuroengineering Group (NBIO), 415-421. doi: 10.5220/0003725704650469

Fondo de la Naciones Unidas para la Infancia (Unicef). (2011). Estado Mundial de la Infancia: la adolescencia una época de oportunidades. Nueva York: Unicef.

Fondo de la Naciones Unidas para la Infancia (Unicef). (2013). Situación Actual con respecto al uso indebido de drogas. Informe de la Secretaria. Viena: Unicef.

Franken, I. H. (2003). Drug craving and addiction: integrating psychological and neuropsychopharmacological approaches. Progress in Neuro-Psychopharmacology and Biological Psychiatry, 27(4), 563-579. doi: 10.1016/S02785846(03)00081-2

Garcia, J. M., Garcia, M., \& Rivera, S. (2015). Potencial resiliente en familias con adolescentes que consumen y no consumen alcohol. Acta Colombiana de Psicología, 18(2), 163-172. doi: 10.14718/ACP.2015.18.2.14

García, L. M., \& Barriguete, B. (2012). Avance en la comprensión del fenómeno de las adicciones. México: Secretaría de Salud.

Gaviria, M. I. (2010). Interacciones neuroquímicas y comportamentales entre 3.4metilenodiosimetanfetamina (MDMA) y etanol: implicación en la neurotoxicidad de MDMA y en el consumo de etanol en roedores (Tesis doctoral). Facultad de Medicina, Universidad Complutense de Madrid, Madrid.

Gilpin, N. G., \& Koob, G. F. (2008). Neurobiology of Alcohol Dependence: Focus on Motivational Mechanisms. Alcohol
Research y Health, 31(3), 185- 195. Recuperado de https:// www.ncbi.nlm.nih.gov/pmc/articles/PMC2770186/pdf/arh31-3-185.pdf

Gobierno Nacional de la República de Colombia (2013). Estudio Nacional de Consumo de Sustancias Psicoactivas en Colombia. Bogotá: ALVI Impresores S.A.S.

Golarai, G., Ghahremani, D. G., Whitfield-Gabrieli, S., Reiss, A., Eberhardt, J. L., De Gabrieli, J., \& Grill-Spector, K. (2007). Differential development of high-level visual cortex correlates with category-specific recognition memory. Nature Neuroscience, 10(4), 512-522. doi: 10.1038/nn1865

González, L., Ortiz, T., Gutiérrez, C., \& González, C. (2011). Estudio conjunto de magnetoencefaografía electroencefalogrfia en epilepsia (Tesis doctoral). Departamento de Psiquiatría. Universidad Complutense de Madrid, Madrid. Recuperado de http://eprints.ucm.es/12536/1/T32720.pdf

Greenwald, M. K., \& Roehrs, T. A. (2005). Mu-opioid Self-Administration vs Passive Administration in Heroin Abusers Produces Differential EEG Activation. Neuropsychopharmacology, 30(1), 212-221. doi:10.1038/sj.npp.1300596

Guardia, J. (2001). Neuroimagen y drogodependencias. Trastornos adictivos, 3(2), 95-110. Recuperado de http://www.elsevier.es/es-revista-trastornos-adictivos-182-pdf-13015408

Heming, R. I., Glover, B. J., Koeppl, B., Phillips, R. L., \& London, E. D. (1994). Cocaine-Induced Increases in EEG Alpha and Beta Activity: Evidence for Reduced Cortical Processing. Neuropsychopharmacology, 11(1), 1-9. doi: 10.1038/npp.1994.30

Jacobus, J., \& Tapert, S. F. (2013). Neurotoxic effects of alcohol in adolescence. Annual Review of Clinical Psychology, 9, 703-721. doi: 10.1146/annurev-clinpsy-050212-185610

Kauhanen, L., Jylanki, P., Lehtonen, J., Rantanen, P., Alaranta, H., \& Sams, M. (2007). EEG-Based Brain-Computer Interface for Tetraplegics. Computational Intelligence and Neuroscience, 2007 (23864), 1-11. doi: 10.1155/2007/23864

Kiyatkin, E. A., \& Smimov, M. S. (2010). Rapid EEG desynchronization and EEG activation induced by intravenous cocaine in freely moving rats: a peripheral, nondopamine neural triggering. American journal of physiology, 298(2), R285-R300. doi:10.1152/ajpregu.00628.2009

Kosten, T. R., \& George, T. P. (2002). The Neurobiology of Opioid Dependence: Implications for Treatment. Science y Practice Perspectives, 1(1), 13-20. doi: 10.1151/spp021113

Kreek, M. J., Levran, O., Reed, B., Schlussman, S. D., Zhou, Y., \& Butelman, E. R. (2012). Opiate addiction and cocaine addiction: underlying molecular neurobiology and genetics. The Journal of Clinical Investigation, 122(10), 3387-3393. doi:10.1172/JCI60390

Lacono, W., Malone, S. M., \& McGue, M. (2008). Behavioral Disinhibition and the Development of Early- Onset Addiction: Common and Specific influences. Annual Review of Clinical Psychology, 4, 325-348. doi: 10.1146/annurev. clinpsy.4.022007.141157 
Lansbergen, M. M., Dumont, G. J., Van Gerven, J. M., Buitelaar, J. K., \& Verke, R. J. (2011). Acuteeffects of MDMA (3.4-methylenedioxymethamphetamine) on EEG oscillations: alone and in combination with ethanol or THC (delta9-tetrahydrocannabinol). Psychopharmacology, 213(4), 745-756. doi: 10.1007/s00213-010-2031-4

Lansbergen, M. M., Arns, M., Van Dongen-Boomsma, M., Spronk, D., \& Buitelaar, J. (2011). The increase in theta/ beta ratio on resting-state EEG in boys with attentiondeficit/hyperactivity disorder is mediated vy slow alpha peak frequency. Progress in Neuro-Psychopharmacology and Biological Psychiatry, 35(1), 47-52. doi: 10.1016/j. pnpbp.2010.08.004

Lee, L. (2013). Mechanisms and Functions of theta Rhythms. Annual Review of Neuroscience, 36, 395-312. doi: 10.1146/ annurev-neuro-062012-170330

León, M. 1., González, L. H., León, A., Armas, J. O., Urquiza, A., \& Rodríguez, G. (2014). Bases Neurobiológicas de la adicción al alcohol. Revista Finlay, 4(1), 40-53. Recuperado de http://www.revfinlay.sld.cu/index.php/finlay/article/ view/253/1257

León, O. G., \& Montero, I. (2003). Métodos de investigación en Psicología y Educación (3. ${ }^{\mathrm{a}}$ ed.). Madrid: Mc Graw-Hill.

Littel, M., Franken, I. H., \& Van Strien, J. W. (2009). Changes in the Electroencephalographic Spectrum in Response to Smoking Cues in Smokers and Ex-Smokers. Neuropsychopharmacology, 59(1), 43-50. doi: 10.1159/000205517

Liu, X., Vaupel, D. B., Grant, S., \& London, E. D. (1998). Effect of Cocaine-Related Environmental Stimuli on the Spontaneous Electroencephalogram in Polydrug Abusers. Neuropsychopharmacology, 19(1), 10-17. doi: 10.1016/ S0893-133X(97)00192-9

Lomas, T., Ivtzan, I., \& Cynthia, H. Y. (2015). A systematic review of the neurophysiology of mindfulness on EEG oscillations. Neuroscience y Biobehavioral Reviews, 57, 401410. doi: 10.1016/j.neubiorev.2015.09.018

López-Caneda, E., Mota, N., Crego, A., Velásquez, T., Corral, M., Rodríguez, S., \& Cadaveira, F. (2014). Anomalías neurocognitivas asociadas al consumo intensivo de alcohol (binge drinking) en jóvenes y adolescentes: una revisión. Adicciones, 26(4), 334-359. doi: 10.20882/adicciones.39

Madoz-Gúríde, A., Ochoa, E., \& Martínez, B. (2009). Consumo de cocaína y daño neuropsicológico: implicaciones clínicas. Revista de Medicina Clínica, 132(14), 555-559. Recuperado de https://www.researchgate.net/publication/246618526_Consumo_de_cocaina_y_dano_neuropsi cologico_Implicaciones_clinicas

Madoz-Gúrpide, A., \& Ochoa-Mangado, E. (2012). Alteraciones de funciones cognitivas y ejecutivas en pacientes dependientes de cocaína: estudio de casos y controles. Revista de Neurología, 54(4), 199-208.

Mena, I., Dorr, A., Viani, S., Neubauer, S., Gorostegui, M. E., Dorr, M. P., \& Ulloa, D. (2013). Efectos del consumo de marihuana en escolares sobre funciones cerebrales demostrados mediante pruebas neuropsicológicas e imágenes de neuro-SPECT. Salud mental, 36(5), 367-374. Recuperado de http://www.scielo.org.mx/pdf/sm/v36n5/ v36n5a3.pdf

Merzenich, M. (2003). Ejercicio “enfoco mi Atención” del Programa virtual de entrenamiento Brain HQ. Recuperado de https://es.brainhq.com/?fblogin=returning_fb\#train/atten tion $/ 0 / 0 / 0$

Moratalla, R. (2008). Neurobiología de la cocaína. Trastornos Adictivos, 10(3), 143-150. Recuperado de http://www.elsevier.es/es-revista-trastornos-adictivos-182-pdf-13128589

Mota, N. G., \& Corral, M. M. (2011). Estudio longitudinal del perfil neuropsicológico del consumo intensivo de alcohol entre jóvenes universitarios (Tesis doctoral). Departamento de Psicología Clínica y Psicobiología, Universidad de Santiago de Compostela, Santiago de Compostela. Recuperado de https://minerva.usc.es/xmlui/bitstream/hand le/10347/3388/9788498876345_content.pdf?sequence $=1$

National Intitute on Drug Abuse (2010). Cocaína: abuso y adicción. España. Recuperado de http://www.ndcrc.org/ content/serie-de-reportes-de-investigaci $\% \mathrm{C} 3 \% \mathrm{~B} 3 \mathrm{n}$ coca $\% \mathrm{C} 3 \%$ ADna-abuso-y-adicci $\% \mathrm{C} 3 \% \mathrm{~B} 3 \mathrm{n}$.

Nestler, E. J. (2005). The Neurobiology of Cocaine Addiction. Science y Practice Perspectives, 3(1) 4-12.

Nicolas, L. F., \& Gómez, J. (2012). Brain Computer Interfaces, a Review. Sensors, 12(2), 1211-1279. doi: 10.3390/ s120201211

Oficina de Naciones Unidas contra la droga y el delito (UNODC). (2016). Informe Mundial sobre las Drogas. Recuperado de https://www.unodc.org/doc/wdr2016/WDR_2016 ExSum_spanish.pdf

Organización Mundial de la Salud. (2011). La prueba de detección de consumo de alcohol, tabaco y sustancias (ASSIST): Manual para uso en la atención primaria. Estados Unidos: OMS.

Pagani, L. S. (2014). Environmental tobacco smoke exposure and brain development: The case of attention déficit/ Hyperactivity disorder. Neuroscience y Biobehavioral Reviews, 44, 195-205. doi: 10.1016/j.neubiorev.2013.03.008

Partilla, J. S., Dempsey, A. G., Nagpal, A, S., Blough, B. E., Baumann, M. H., \& Rothman, R. B. (2006). Interaction of Amphetamines and Related Compounds at the Vesicular Monoamine Transporter. The journal of pharmacology and experimental therapeutics, 319(1), 237-246. doi: 10.1124/ jpet.106.103622

Pérez-Rubio, G., Urdapilleta, E., Camarena, A., ReséndizHernández, J. M., Méndez, M., ... Falfán-Valencia, R. (2011). Visión general de la neurobiología y genética en la adicción a la nicotina. Neumología y cirugia del Tórax, 70(3), 179-187. Recuperado de http://www.medigraphic. com/pdfs/neumo/nt-2011/nt113g.pdf

Phillips, R. L., Heming, R., \& London, E. D. (1994). Morphine Effects on the Spontaneous Electroencephalogram in Polydrug Abusers: Correlations with Subjective Self-Reports. 
Neuropsychopharmacology, 10(3), 171-181. doi: 10.1038/ npp.1994.19

Quesada, M. E., Diaz-Pérez, G. F., Herrera, M., TamayoPorras, M., \& Rubio-López, R. (2007). Características del electroencefalograma cuantitativo y trastorno cognitivos en pacientes alcohólicos. Revista de Neurología, 44(2), 81-88. Recuperado de https://dialnet.unirioja.es/servlet/ articulo?codigo $=2232935$

Redolar, D (2008). Cerebro y adicción. Barcelona: Editorial UOC.

Reid, M. S., Flammino, F., Howard, B., Nilsen, D., \& Prichep, L. S. (2006). Topographic Imaging of Quantitative EEG in Response to Smoked Cocaine Self-Administration in Humans. Neuropsychopharmacology, 31, 872-884. doi:10.1038/ sj.npp. 1300888

Ríos, M., Muñoz, J., \& Paúl, N. (2007). Alteraciones de la atención tras daño cerebral traumático: evaluación y rehabilitación. Revista de Neurología, 44(5), 291-297. Recuperado de http://www.sld.cu/galerias/pdf/sitios/rehabilitacion-adulto/ alteraciones_de_la_atencion_tras_dano.pdf

Rodríguez, U., Carrillo, E., \& Soto, E. (2005). Cannabinoides: neurobiología y usos médicos. Elementos: Ciencia y Cultura, 12(60), 3-9. Recuperado de http://www.redalyc.org/ pdf/294/29406001.pdf

Rosselli, M., \& Ardila, A. (1996). Cognitive effects of cocaine and polydrug abuse. Journal of Clinical and Experimental Neuropsychology, 18(1), 122-135. doi: 10.1080/01688639608408268

Sánchez, A., Vázquez, C., \& Valiente, C. (2011). Atención selectiva como mecanismo de regulación emocional y factor de vulnerabilidad a la depresión (Tesis doctoral). Facultad de Psicología, Universidad Complutense de Madrid, Madrid. Recuperado de http://eprints.ucm.es/14460/1/T33365.pdf

Santana, D., Ramírez, M., \& Ostrosky-Solís, F. (2004). Novedades en tecnología de la rehabilitación: una revisión acerca de la interfaz cerebro-computadora. Revista de Neurología, 39, 447-450. Recuperado de https://www.researchgate. net/profile/Maura_Ramirez/publication/221657835_Novedades_en_tecnōlogia_de_la_rehabilitacion_Unā_revision_acerca_de_interfaz_cerebro-computadora/ links/0 $\overline{\mathrm{d}} 1 \mathrm{c} 84 \mathrm{f54 \textrm {efc }} 8 \mathrm{ad} 4 \mathrm{aa} 000000 /$ Novedades-en-tecnologia-de-la-rehabilitacion-Una-revision-acerca-de-interfazcerebro-computadora.pdf
Salgado, A. (2003). Evaluación psicofisiológica: Introducción a la Psicofisiología. España: Ed. Universidad Pontificia de Salamanca.

Silva, J. (2011). Métodos en neurociencias cognitivas. México: Manual Moderno.

Squire, R. F., Noudoost, B., Schafer, R. J., \& Moore, T. (2013). Prefrotal Contributions to visual selective attention. Annual Review of Neuroscience, 36, 451-466. doi: 10.1146/ annurev-neuro-062111-150439

Talamillo, T. (2011). Manual básico para enfermeros en electroencefalografía. Enfermería Docente, 94, 29-33. Recuperado de http://www.juntadeandalucia.es/servicioanda luzdesalud/huvvsites/default/files/revistas/ED-094-07.pdf

Urigüen, L., \& Callado, L. F. (2010). Cocaína y cerebro. Trastornos Adictivos, 12(4), 129-134. doi: 10.1016/S15750973(10)70025-8

Van Hell, H. H., Vink, M., Ossewaarde, L., Jager, G., Kahn, R. S., \& Ramsey, N. F. (2011). Efectos crónicos del consumo de cannabis sobre el sistema de recompensa humano: un estudio de RMf. Psiquiatría Biológica, 18(2), 45-54. doi:10.1016/j.psiq.2011.08.003

Weise, M., Eisenhofer, G., \& Merke, D. P. (2002). Pubertal and Gender-Related Changes in the Sympathoadrenal System in Healthy Children. The Journal of Clinical Endocrinology y Metabolism, 87(11), 5038-5043. doi: 10.1210/jc.20020205905038

Zhao, Q., \& Zhang, L. (2007). Temporal and Spatial Features of Single-Trial EEG for Brain-Computer Interface. Computational Intelligence and Neuroscience. 1-14. doi: $10.1155 / 2007 / 37695$ 